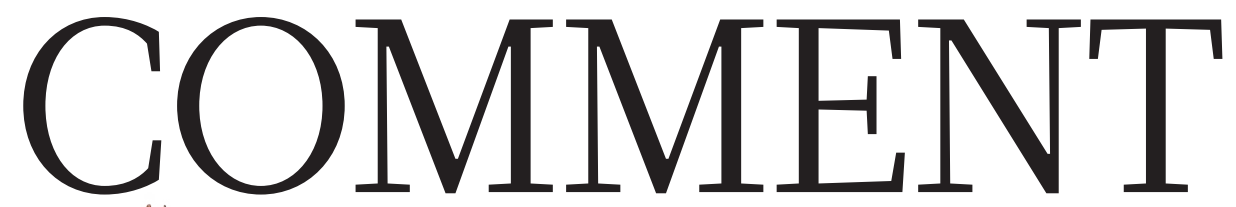

\begin{tabular}{|c|c|c|c|}
\hline $\begin{array}{l}\text { GEOSCIENCE Enough talk - it } \\
\text { is time for action on a global } \\
\text { Earth observatory p.21 }\end{array}$ & $\begin{array}{l}\text { CULTURE This year's must-see } \\
\text { films, exhibitions, music } \\
\text { and events } \mathbf{p} .24\end{array}$ & $\begin{array}{l}\text { CONSERVATION Poaching of fish } \\
\text { in Lake Victoria is a threat to } \\
\text { food and jobs p.27 }\end{array}$ & $\begin{array}{l}\text { POLITICS European academies } \\
\text { flag Brexit's impact on } \\
\text { science in Europe p.27 }\end{array}$ \\
\hline
\end{tabular}

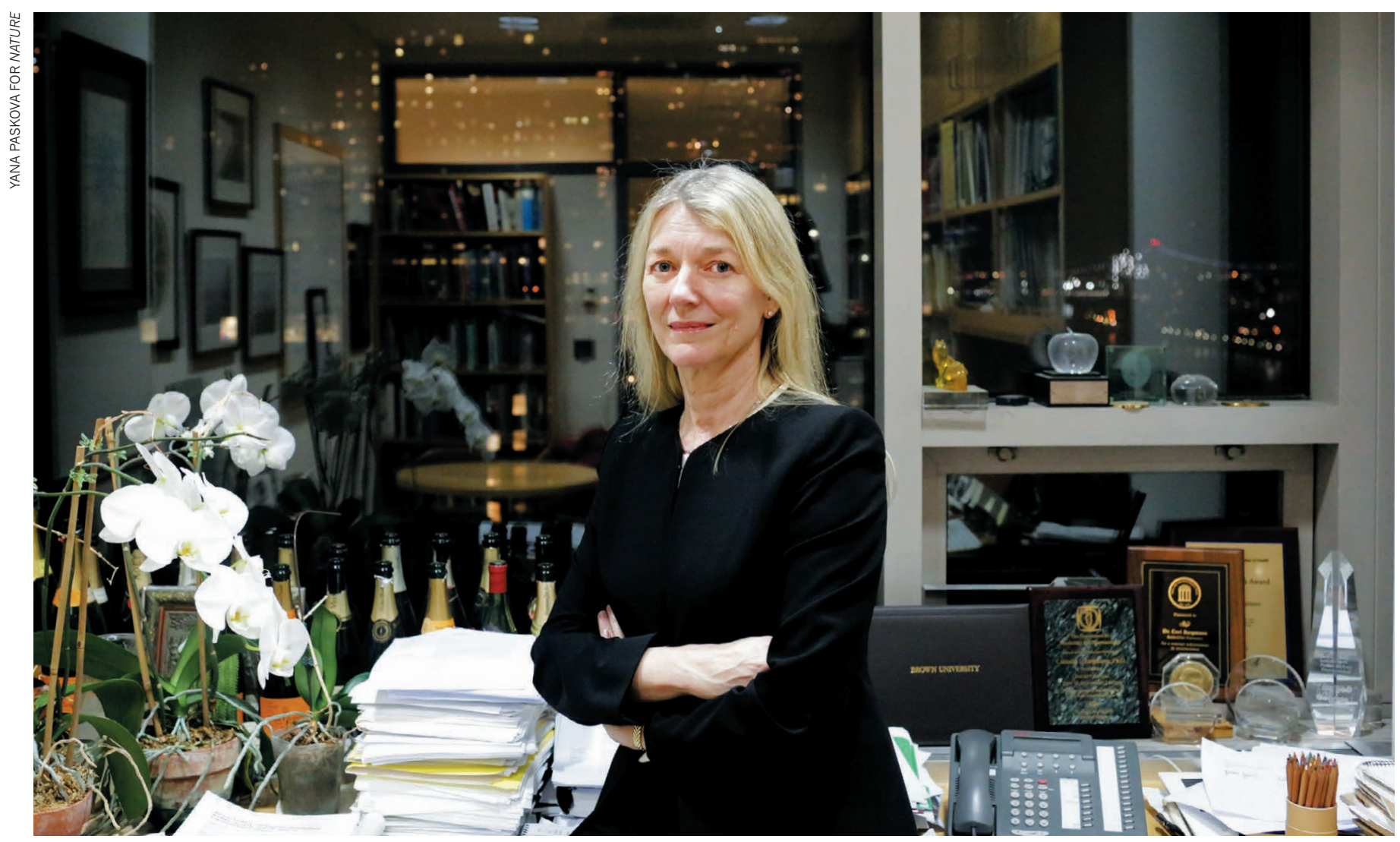

Cori Bargmann heads the Chan Zuckerberg Science Initiative, a philanthropic effort launched in late 2016 to support biomedical research.

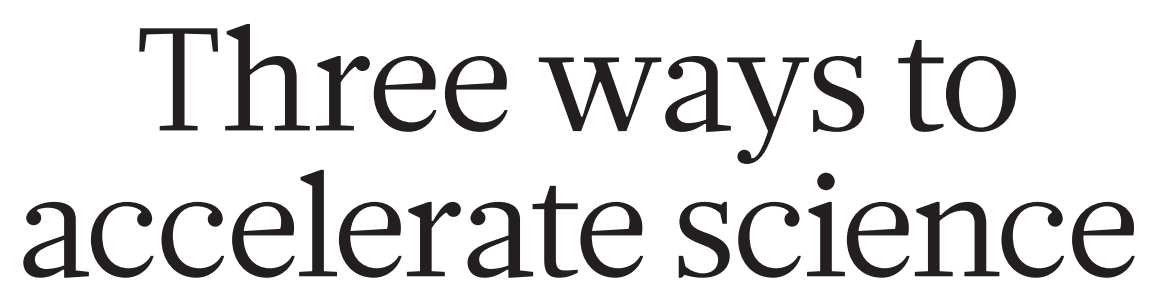

Chan Zuckerberg Science will prioritize the elements that made roundworm studies soar - creativity, openness and shareable resources, writes its president, Cori Bargmann.

I n 1987, I joined the lab of Robert Horvitz at the Massachusetts Institute of Technology in Cambridge as a postdoctoral fellow. I was fascinated by the idea of using genetics to probe the neural basis of behaviour. And a unique resource drew me to the tiny transparent worm Caenorhabditis elegans: a wiring diagram of the 302 neurons in the adult worm's nervous system.

Work led by John White, then a
C. elegans researcher at the Medical Research Council's Laboratory of Molecular Biology (LMB) in Cambridge, UK, had mapped all the connections between the worm's neurons by slicing the animal into thousands of sections and tracing each cell using electron microscopy. This wiring diagram, combined with the worm's short life cycle of a few days, offered a tremendous opportunity to relate the development and function of the nervous system to genes and neurons. And it was just one of the many shared resources available for C. elegans research.

The findings made using C. elegans have been remarkable. Among these are the caspase system that controls programmed cell death; the netrin system that guides neuronal connectivity; and the post-transcriptional gene-regulatory 
$>$ pathways involving microRNAs and small interfering RNAs.

I believe that the success of these projects emerged in part from a unique research culture and infrastructure. Now I want to help put in place similar opportunities on a larger scale, as president of the Chan Zuckerberg Science Initiative, a philanthropic effort launched in late 2016 to support biomedical research.

\section{THE THREE INGREDIENTS}

What made the C. elegans field successful?

A common reference. By the mid1960s, fruit flies and yeast had already been studied for decades. But biologist Sydney Brenner, then at the LMB, wanted to develop a new model organism for studying the big questions in development and neuroscience. He picked C. elegans.

The LMB group began realizing Brenner's goal by developing a shared infrastructure. Brenner and his $\mathrm{PhD}$ student Jonathan Hodgkin created genetic tools, such as strains of worms with wellcharacterized mutations, and mapped the functions of hundreds of genes. Biologist John Sulston led a team that described the complete lineage of all cells, documenting every step in the transformation of a singlecell embryo to the adult worm (J. E. Sulston et al. Dev. Biol. 100, 64-119; 1983). White, Brenner and their team mapped the connections of all of the worm's neurons, naming every neuronal cell and mapping its lineage and place in the circuit.

Descriptive science - observing, recording, describing and classifying phenomena - is often valued less than hypothesis testing. But the common resources that result help everyone. Every experiment I have done has been grounded in White and colleagues' wiring paper, affectionately known as The Mind of a Worm (J. G. White et al. Phil. Trans. R. Soc. Lond. B 314, 1-340; 1986).

The success of these projects, and the recognition of their value by the community, meant that it was easy to convince C. elegans researchers of the worth of the first genome projects discussed in the 1990s. They were similarly game for making and sharing the first RNAi libraries (collections of small interfering RNAs for disrupting gene function, matched to every gene in the worm's genome), the Wormbase organismal database (a repository of everything that's known about $C$. elegans biology) and, more recently, the global genetic-diversity resource CeNDR (www. elegansvariation.org).

Creative exploration. Today, people are often encouraged to stay in a research niche for long stretches of their careers - to learn 'more and more about less and less'. One effect of this is that students stay in the same

fields as their advisers, and both learn less than they might have done had they diversified.

By contrast, the MRC mavens took a gamble that there were many interesting questions left in biology, and that buying lots of lottery tickets - in the form of different research areas - would pay off for the success and prestige of the field. Thus, there was a conscious decision among those involved in the foundational work on C. elegans to maximize discovery by encouraging people to explore the worm's biology widely. When I joined his lab, Horvitz told me I could study any problem that could be addressed in a worm.

Openness. Today, two concerns tend to come up in discussions about releasing findings before their formal publication: is the work accurate, and will people steal the results?

When I started working on C. elegans, people published in a semiregular newsletter called the Worm Breeder's Gazette $(W B G)$. Most of the groups that were using the worm as a model organism published in every issue; the one-page abstracts typically described a single result. The $W B G$ was fast. A few weeks or months after you had a result,

\section{"We want all of biomedical science to be faster, more robust, sharable and scalable."} it would be out there for everyone to see. In fact, some $W B G$ abstracts preceded papers by five years or more.

Some of the findings reported in the $W B G$ didn't hold up long-term. And that was okay; results that can't be replicated soon get ignored. As for stealing others' work, I think that the very openness of the C. elegans field acted as a deterrent.

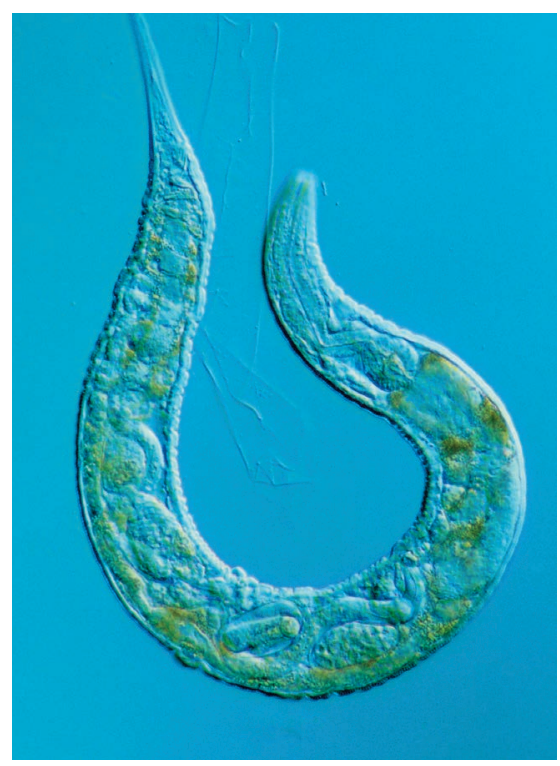

The roundworm Caenorhabditis elegans.
Everyone knew what was in the $W B G$, and there was a clear expectation that if you used someone else's result, you included that person in your study or cited them. The scientists who read the $W B G$ were the same ones who were going to review your grants, papers and case for promotion, so the implicit requirement to respect that culture had teeth. In many cases, the openness seemed to relieve tensions; people could find out in advance whether similar work was in progress in another lab, and coordinate publications.

\section{SHAPING SCIENCE TODAY}

The mission of the Chan Zuckerberg Science Initiative, founded in 2016 by Mark Zuckerberg and Priscilla Chan, is to support science and technology that will make it possible to cure, prevent or manage all diseases by the end of the century. It's a bold goal. But the end of the century is still 82 years away. Going back in time a similar distance, much of modern medicine would have been unthinkable - from organ transplants and deep brain stimulation to treating cancer by manipulating the immune system.

All of these advances were built on a foundation of basic biomedical science. To enable the next generation of discoveries, we at the Chan Zuckerberg Initiative want all of biomedical science to be faster, more robust, sharable and scalable. We're starting a number of different programmes - both locally and globally - to try out ideas for accelerating science and driving collaboration.

First, we want to support scientific infrastructure projects that change the landscape for research fields. In collaboration with other groups and funders, we are supporting the Human Cell Atlas (HCA), an endeavour to map all the cells in the human body. For the trillions of cells that make up the human body, we don't know how many cell types there are, nor their exact numbers, locations, molecular compositions and spatial relationships in tissues and organs. Such knowledge could benefit all biologists who study humans.

In addition to funding experimental scientists working on the HCA, the Chan Zuckerberg Initiative is funding external collaborators and an in-house group of software engineers and computational biologists focused on developing new data platforms and tools for biomedical science. This is an opportunity, because many of the advances in technology that have happened in the commercial sector have not been available to academic science. As a neuroscientist, I take this personally: numerous recent innovations in machine learning and neural networks originated in neuroscience, so biologists should be able to share the benefits. 
Second, to foster creativity, we plan to support people who want to work in new areas - especially young researchers setting up their own labs. Most scientists do their most creative work at this early stage of their careers. But - understandably — it's often hard to obtain funding unless you can demonstrate expertise in a particular area. The Chan Zuckerberg Initiative could fill a niche by taking on more risks than other funders. That risk is worthwhile if it brings people into biomedical areas in which the need is great but current research is narrowly directed. Unfortunately, disease-relevant fields can be some of the hardest to break into for someone with a new idea or approach. Certain disease foundations, such as the Hereditary Disease Foundation for Huntington's disease or the Simons Foundation Autism Research Initiative, have done this well in the past. But we think that there is room to scale up this model to many other biomedical problems.

Finally, on openness. We believe that research advances when people build on each others' work. So our principles include making data, protocols, reagents and code freely available for other scientists to use. As an example of this approach, the HCA has committed to making its reference data publicly available after quality-control checks. Indeed, the Chan Zuckerberg Initiative engineering team and our HCA collaborators are building all of the software for the 'data coordination' arm of the project on the open-source platform Github.

We're also supporting external groups that share these values and goals. For instance, we're funding bioRxiv, the largest and fastest-growing preprint repository for the biological sciences - and a leader in bringing biology towards the level of sharing that's expected in the physical and computer sciences.

The Chan Zuckerberg Initiative is just starting, and we have a lot to learn. But I've been lucky to work in areas in which the free exchange of ideas and results is the norm. In my experience, such an approach creates the most dynamic fields. Now I have the chance to lead a new funding venture, and to explore whether openness or dynamism comes first. After all, as scientists we do experiments; as funders, we can do experiments too. $\square$

Cori Bargmann is president of science at the Chan Zuckerberg Initiative in Palo Alto, California; and professor of genetics, neuroscience and behaviour at the Rockefeller University, New York, USA. e-mail:science@chanzuckerberg.com

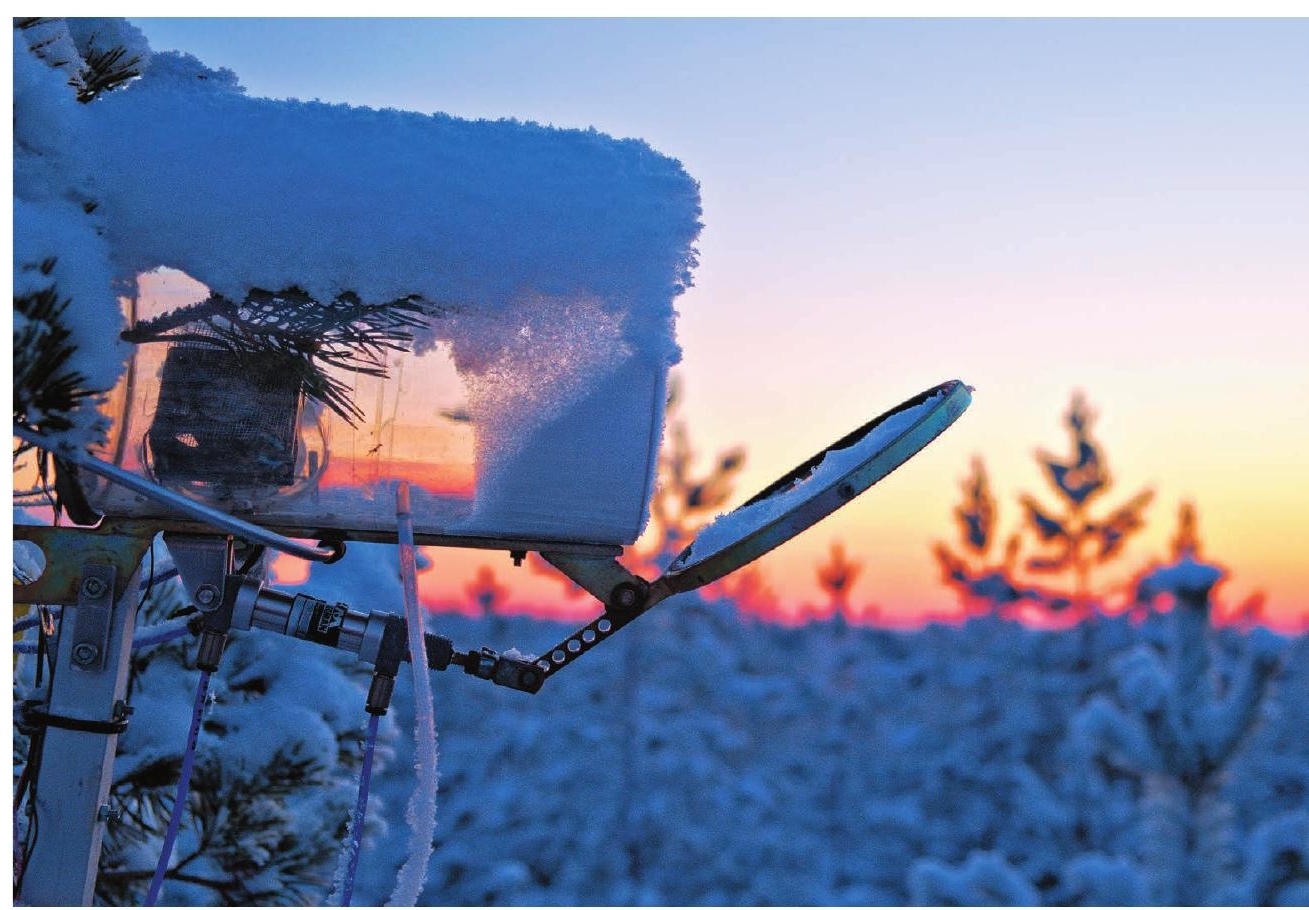

An enclosure for measuring gas exchange between plants and the atmosphere at a station in Finland.

$$
\text { Build a global }
$$

Earth observatory

Markku Kulmala calls for continuous, comprehensive

$$
\begin{gathered}
\text { monitoring of interactions between the planet's } \\
\text { surface and atmosphere. }
\end{gathered}
$$

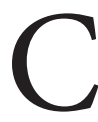

limate change. Water and food security. Urban air pollution. These environmental grand challenges are all linked, yet each is studied separately.

Interactions between Earth's surface and the atmosphere influence climate, air quality and water cycles. Changes in one affect the others. For example, increasing carbon dioxide enhances photosynthesis. As they grow, plants withdraw greenhouse gases from the atmosphere, but they also release volatile organic compounds such as monoterpenes. These speed up the formation of aerosol particles, which reflect sunlight back into space. Our actions - such as emission-control policies, urbanization and forestry - also affect the atmosphere, land and seas ${ }^{1-5}$.

Satellites and stations on the ground track greenhouse gases, ecosystem responses, particulate matter or ozone independently of each other. Coupled observations are occasionally performed, but in intensive bouts. Vast areas of the globe - including Africa, eastern Eurasia and South America - are barely sampled.
The result is a cacophony of information that yields little insight. It is like trying to forecast weather in November with spotty measurements of rain, wind, temperature or pressure from June.

The answer is a global Earth observatory - 1,000 or more well-equipped ground stations around the world that track environments and key ecosystems fully and continuously. Data from these stations would be linked to data from satellite-based remote sensing, laboratory experiments and computer models.

Researchers could find new mechanisms and feedback loops ${ }^{6}$ in this coherent data set. Policymakers could test policies and their impacts. Companies could develop environmental services. Early warnings could be provided for extreme weather, and quick responses initiated during and just after chemical accidents.

A global observatory has been discussed for more than a decade, but is only now feasible ${ }^{7}$. Instruments have matured; for example, today's mass spectrometers 